\title{
Changes in absolute detection threshold and in subjective intensity of suprathreshold stimuli during olfactory adaptation and recovery ${ }^{1}$
}

\author{
GORDON T. PRYOR, GERALD STEINMETZ, AND HERBERT STONE ${ }^{2}$ \\ STANFORD RESEARCH INSTITUTE
}

\begin{abstract}
Olfactory adaptation and recovery to methyl isobutyl ketone at a concentration 10 times the absolute detection threshold $\left(I_{t_{o}}\right)$ was intensively studied in two human Ss. A combined psychophysical procedure was used that allowed comparisons of changes in threshold $\left(I_{t}\right)$ with changes in the subjective intensity of suprathreshold stimuli. Information was also obtained on the effect of the adapting stimulus on the psychophysical power function for this odorant. A threshold detection procedure was used to estimate changes in $I_{t}$; an unstructured magnitude-estimation procedure was used to monitor changes in the subjective intensity of suprathreshold stimuli and the psychophysical power function. The data provided additional information on the behavioral course of olfactory adaptation and recovery and suggested that this combined method can be used profitably for further investigations of this kind. Complementary to the work of Cain and Engen (1969), the results suggested an increase in the exponent of the power function with increasing adaptation.
\end{abstract}

Olfactory adaptation is characterized by a marked reduction in sensitivity that may be more or less specific to a particular odor stimulus. Removal of the stimulus allows recovery to previous levels of sensitivity. Two aspects of the process have received independent attention: (1) the change in absolute detection threshold $\left(I_{t}\right)$ (Stuiver, 1958; Steinmetz et al, 1970), and (2) the change in subjective intensity of the adapting stimulus over time (Ekman et al, 1967; Steinmetz et al, 1970). Recently, Cain and Engen (1969) have reported the effect of an adapting stimulus on the psychophysical power function; this approach has the advantage of describing a greater portion of the olfactory process. However, they did not attempt to relate their data to changes in $I_{t}$, and only a narrow range (6 to $30 \mathrm{sec}$ ) of adaptation times was studied.

In an earlier study of adaptation and recovery (Steinmetz et al, 1970), we attempted to compare changes in $I_{t}$ with changes in perceived intensity of the adapting stimulus. A threshold-detection procedure similar to that described by Stuiver (1958) was used to monitor changes in $I_{t}$, and a modified category scale similar to that described by Stone (1966) was used to monitor changes in perceived intensity. The two methods were employed separately but with the same Ss. Adaptation was monitored until asymptote was reached, followed by recovery.

Although the two procedures yielded systematic qualitative information about the adaptation-recovery process, a direct quantitative comparison between changes in $I_{t}$ and in subjective intensity was not considered fully justified due to possible artifacts inherent in both procedures. The change in $I_{t}$ during adaptation was undoubtedly influenced by the occurrence of some recovery when the adapting stimulus was interrupted for insertion of test trials. The category-rating procedure suffers from possible response bias, discontinuous and limited choices of response, and the need to rely on sensorial memory for the initial standard over long periods of time.

The present experiment was conducted in an effort to obtain a more direct and more complete description of the effect of an adapting stimulus on $I_{t}$ and on the intensity of suprathreshold stimuli as a function of time, and recovery in sensitivity in the absence of the adapting stimulus. To overcome the objection raised about the threshold-detection procedure, only one test trial was given after a preselected period of adaptation during a session. To overcome the objections to the category-rating procedure, Ss were trained t o use an unstructured magnitude-estimation procedure for which they developed (prior to the experiment) an internal scale that did not depend on a particular reference standard. Finally, the subjective intensities of several suprathreshold concentrations were determined as a function of adaptation and recovery in order to monitor changes in the psychophysical power function. All concentrations of test stimuli, including the adapting stimulus, were calculated in multiples of $I_{t}$ under nonadapted conditions $\left(\mathrm{I}_{\mathrm{t}_{\mathrm{o}}}\right)$.

\section{Subjects \\ METHODS \\ Two Ss, one male and one female, were} drawn from a larger pool for intensive testing in these experiments.

\section{Apparatus}

An olfactometer was used to present the stimuli. The original apparatus has been described elsewhere (Stone \& Bosley, 1965). In brief, it is an air-dilution system that permits delivery of a known concentration of an odorant for a controlled duration. In these experiments the apparatus had been modified to increase the efficiency of its operation (Stone et al, 1969). The modifications consisted of the addition of electronic timers and solenoid valves to facilitate ease of odor presentation and to select odor intensities and test intervals more rapidly and accurately. Two channels were used to present the stimuli; one supplied the adapting stimulus $\left(10 \times I_{t_{0}}\right)$ and the other was used to present test stimuli. Both entered the main air stream just before its outlet to $S$. Communication between $E$ and $S$ during testing was by a visual system of light signals and by $S$ 's vocal report of magnitude estimation.

Concentrations of the stimuli were calculated from vapor pressure, experimental temperature, and gas-flow rates, according to previously established procedures (Stone et al, 1962).

\section{Test Odorant}

The odorant used was methyl isobutyl ketone. Purity, checked by gas-chromatographic analysis, was $>99 \%$.

\section{Experimental Design}

$\mathbf{I}_{t_{0}}$ was determined prior to the experiment and was verified at the beginning of each test session (Steinmetz et al, 1969). A scale of subjective intensity for the odorant was then established for each $S$, using unstructured magnitude estimation (see below). The scales were verified at the conclusion of the experiment. A series of sessions was then run in which an adapting stimulus of $10 \times \mathrm{I}_{\mathrm{t}_{\mathrm{o}}}$ was presented for $25,50,100$, 200,400 , or $800 \mathrm{sec}$, followed by recovery. The duration of the adapting stimulus was varied randomly from session to session to minimize response bias. Six to 10 sessions were completed for each $S$ for each adaptation period. One or two sessions were completed on any particular 


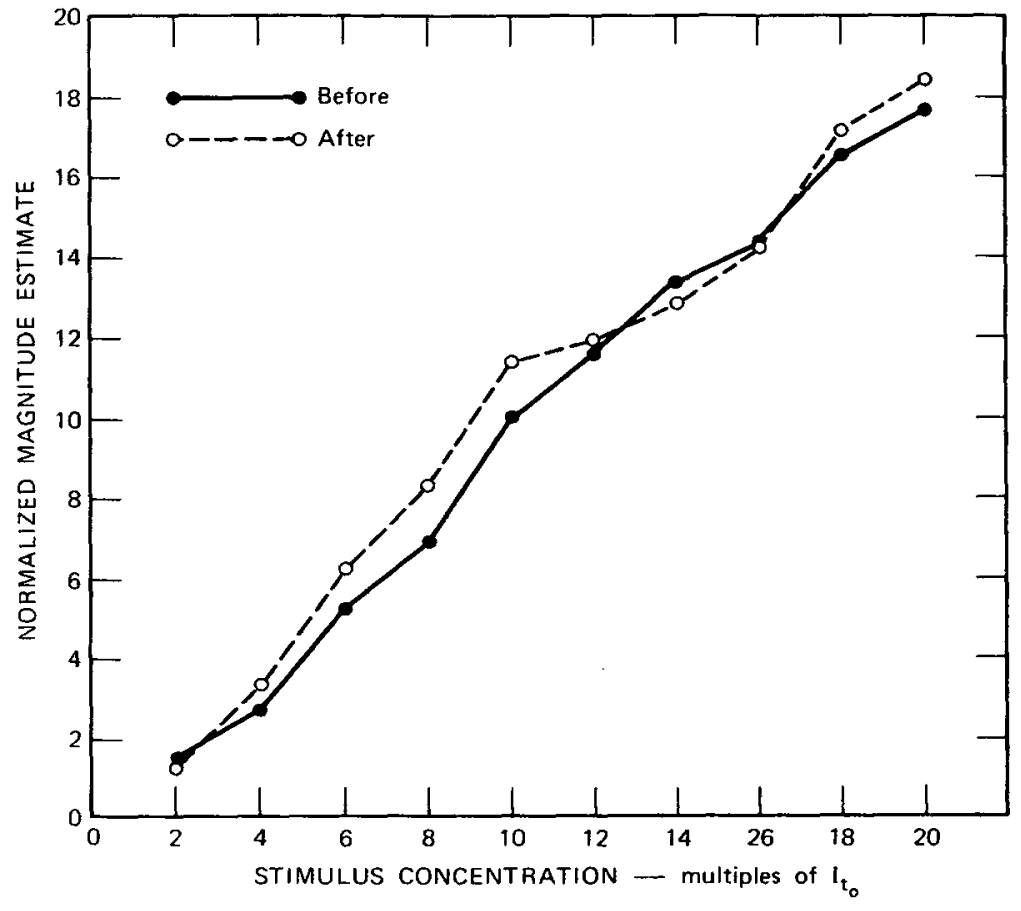

Fig. 1. Average normalized scales of subjective intensity for the odorant methyl isobutyl ketone for two Ss before and after the adaptation-recovery phase of the experiment.

day for each $\mathrm{S}$, several times a week.

\section{Magnitude Estimation}

Each $S$ was instructed to assign any convenient number to his subjective estimate of the intensity of a series of 10 stimuli. The stimuli were equal multiples of $I_{t_{0}}$ ranging from 2 to 20 and were presented randomly for $5 \mathrm{sec}$, with an intertrial interval of $25 \mathrm{sec}$. No standard was used, since it was intended for each $S$ to develop a reliable internal scale that would not depend on memory for any one comparison stimulus. Also, no instructions as to the numbers to be assigned were given. Eleven to 16 sessions were given prior to the adaptation-recovery phase of the experiment, and 5 sessions were given afterwards as a check.

\section{Adaptation-Recovery}

An adaptation-recovery session was conducted as follows. The adapting stimulus was turned on for a preselected period. At the onset of the adaptation period $S$ was instructed to rate the intensity of the adapting stimulus as described above. Another estimate was requested during the last $5 \mathrm{sec}$ of the adaptation period. Then the adapting stimulus was removed and, after $2 \mathrm{sec}$, a test stimulus was presented until $S$ responded or for $5 \mathrm{sec}$. S's response at this time consisted of whether or not he could subjective intensity of suprathreshold stimuli. The suprathreshold stimuli used isobutyl ketone and subsequent recovery. were 4,10 , or 20 multiples of $I t_{0}$. Recovery of It and of subjective intensity was then monitored for a period equal to the duration of the adaptation period. This was done by removing the adapting stimulus and testing periodically with some multiple of $\mathbf{I}_{t_{0}}$. S's response again consisted of whether or not he could detect the test stimulus and, if so, its intensity. Blank samples were inserted periodically to check for "false positives."

\section{RESULTS}

The Psychophysical Function

The two $\mathrm{Ss}$ in this experiment used different moduli in establishing their scales of subjective intensity. One used numbers from 0 to 20 and the other used numbers from 0 to 100 . Nevertheless, the exponents of their respective power functions $\left(\Psi=k_{\varphi}\right)^{n}$ were similar. Prior to the adaptation-recovery phase of the experiment, the exponents were 1.18 and 0.97 . At the conclusion of the experiment they were 1.13 and 1.07 . In order to present the averaged data in comparable form, each S's estimates were normalized to a modulus of 10 corresponding to a concentration of $10 \times \mathrm{I}_{\mathrm{t}_{0}}$ established before the adaptation-recovery phase. The averaged psychophysical functions before and after the main experiment are shown in Fig. 1. There was a tendency to assign slightly higher values to the stimuli after the adaptation-recovery phase of the experiment than before. The possible influence of the adapting stimulus, which was assigned a value and could be considered an anchor point at the



Fig. 2. Average change in $I_{t}$ as a function of self-adaptation to $10 \times I_{t_{0}}$ of methyl 


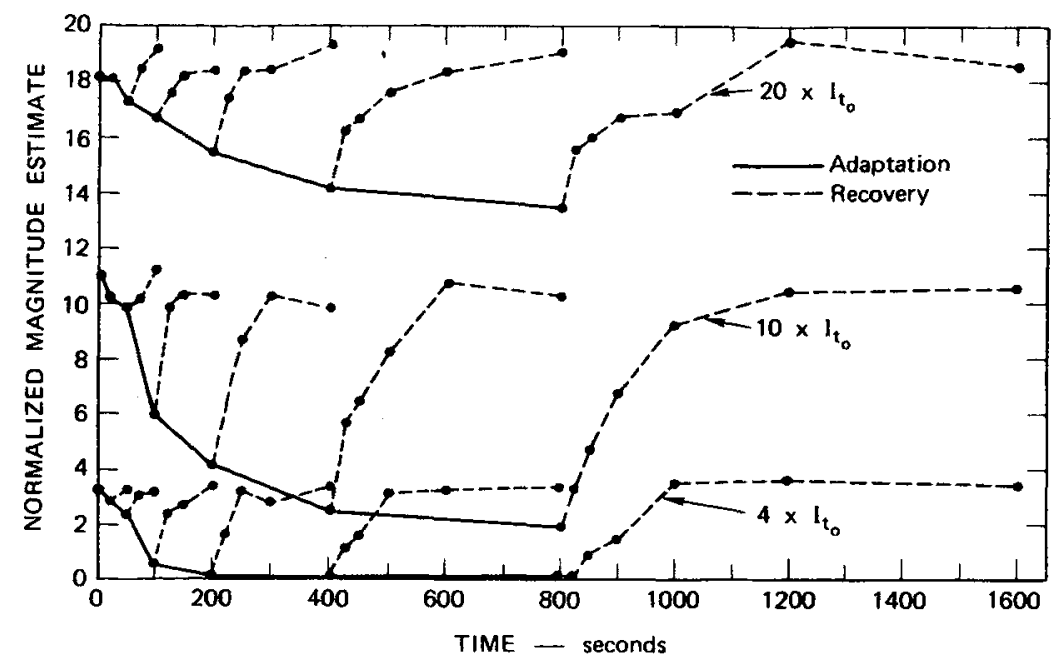

Fig. 3. Average, normalized magnitude estimates of 4,10 , and $20 \times I_{t_{0}}$ as a function of self-adaptation to $10 \times I_{t_{0}}$ of methyl isobutyl ketone and subsequent recovery.

beginning of each adaptation-recovery session, must therefore be recognized. The averaged exponents of the power function before and after the main experiment were $1.08 \pm 0.10$ and $1.10 \pm 0.03$, respectively.

Changes in $I_{t}$ as a Function of Adaptation and Recovery

As shown in Fig. 2, both Ss showed an orderly, nonlinear increase in $I_{t}$ as a function of adaptation time. Since the figure shows the average of the two Ss, it should be pointed out that each adapted at a somewhat different rate. One $S$ reached complete adaptation at the end of $800 \mathrm{sec}$ of adaptation (i.e., $I_{t}=10 \times I_{t_{o}}$ ), whereas the other did not $\left(I_{t}=8.2 \times I_{t_{0}}\right)$. Nevertheless, the shapes of both functions were very similar and were, therefore, combined for the purposes of this report.

Two aspects of these data deserve special comment. First, recovery in $I_{t}$ from long adaptation periods (greater than $100 \mathrm{sec}$ ) appeared to proceed more rapidly than did adaptation to this intensity of the adapting stimulus. Second, recovery time became independent of adaptation time after about $400 \mathrm{sec}$.

Changes in Subjective Intensity of Suprathreshold Stimuli as a Function of Adaptation and Recovery

Figure 3 shows the course of adaptation and recovery for stimuli that were 4,10 , or 20 multiples of $\mathbf{I}_{t_{0}}$. The adaptation curves for $10 \times I_{t_{0}}$ were obtained from estimates given during the last $5 \mathrm{sec}$ of the adaptation period and were normalized to the average estimates given during the first 5 sec. Data for $10 \times I_{t_{0}}$ obtained during the actual test period produced qualitatively similar curves. Only the former are shown since the latter were based on fewer estimates; the discrepancies could reasonably be attributed to small-sample errors.

The subjective intensity of a stimulus $4 \times I_{t_{0}}$ declined to zero within $200 \mathrm{sec}$, whereas the subjective intensity of the adapting stimulus was still above zero after $800 \mathrm{sec}$. As pointed out earlier, one $S$ indicated an $I_{t}=10 \times I_{t_{o}}$ (complete adaptation) after $800 \mathrm{sec}$. However, both Ss reported that they could still perceive the adapting stimulus up to the point when it was turned off. The reasons for this discrepancy are not clear at present. The subjective intensity of a stimulus $20 \times \mathbf{t}_{t_{0}}$, which was initially rated at 18.1 on the normalized scale, declined to 13.7 after $800 \mathrm{sec}$. Recovery of subjective intensity was rapid and appeared to be complete after 200 to $400 \mathrm{sec}$, even for the longest adaptation times. There was a consistent tendency to assign estimates higher than those originally used to the $4 X$ and $20 \times I_{t_{0}}$ stimuli during the final stages of recovery.

Comparison of Changes in $I_{t}$ and Subjective Intensity of Suprathreshold Stimuli as a Function of Adaptation and Recovery

To compare changes in $I_{t}$ with changes in subjective intensity of suprathreshold stimuli, the results were converted to percentage adaptation. This was done as follows. For the threshold detection procedure, $I_{t}$ minus 1.0 was divided by 9 and multiplied by $100\left(I_{t}=1 \times I_{t_{o}}\right.$ was considered $0 \%$ adaptation and $I_{t}=10 \times I_{t_{o}}$ was taken at $100 \%$ adaptation). For the normalized magnitude estimates, the values obtained prior to the main experiment or (for $10 \times I_{t_{o}}$ ) at the beginning of the adaptation period were taken as $0 \%$ adaptation, and the estimates obtained thereafter were experessed as a percentage of these values. These derived values are shown in Fig. 4.

The upper left graph shows the course of adaptation expressed as percentage adaptation for $I_{t}$ and subjective intensity as a function of $\log _{2}$ time. The increase in $I_{t}$ proceeded almost linearly (although slightly concave upwards) up to $800 \mathrm{sec}$. The changes in subjective intensity of suprathreshold stimuli, on the other hand, appeared to proceed as a sigmoid function of $\log _{2}$ time. The subjective intensity of the $20 \times I_{t_{o}}$ stimulus was not appreciably altered up to $25 \mathrm{sec}$, after which a slow, almost linear, decrease was seen up to $400 \mathrm{sec}$. It is likely that asymptote was being approached by $800 \mathrm{sec}$ for this intensity of the adapting stimulus. For $10 \times I_{t_{0}}$ there was a slow decrease in subjective intensity up to $50 \mathrm{sec}$ followed by a rapid decrease and an approaching asymptote by $400 \mathrm{sec}$. The subjective intensity of $4 \times I_{t_{0}}$ proceeded in a similar, but more rapid, fashion to complete adaptation after $400 \mathrm{sec}$.

Recovery is shown for each adaptation time separately in the rest of Fig. 4. Recovery of the subjective intensity of $10 \times I_{t_{o}}$ proceeded more slowly than the recovery of $I_{t}$ after adaptation times of $200 \mathrm{sec}$ and longer. At shorter adaptation times, the subjective intensity of $10 \times I_{t_{0}}$ recovered at comparable rates to $I_{t}$ within the limits of error of this experiment. In these figures the "overshoot" in subjective intensity during recovery is more clearly seen for $4 \times$ and $20 \times I_{t_{0}}$. No such shift was apparent for $10 \times I_{t_{o}}$. In fact, except following adaptation for $50 \mathrm{sec}$, the subjective intensity of $10 \times \mathrm{I}_{\mathrm{t}_{0}}$ did not reach the preadaptation level.

The shapes of the recovery functions were somewhat different from those seen during adaptation. At first, $I_{t}$ recovered more rapidly than it had declined during adaptation and appeared to be more nonlinear in this respect. Similarly, the recovery curves for subjective intensity lacked the sigmoid features seen during adaptation.

\section{Psychophysical Power Functions During Adaptation}

Figure 5 shows the data expressed as psychophysical power functions during adaptation in log-log coordinates. The curve for the nonadapted condition was taken as the average of the curves obtained before and after the adaptation-recovery phase of the experiment. Although in each scale too few points are available to draw firm quantitative conclusions (for this reason the functions were not fitted mathematically), it is clear that the 

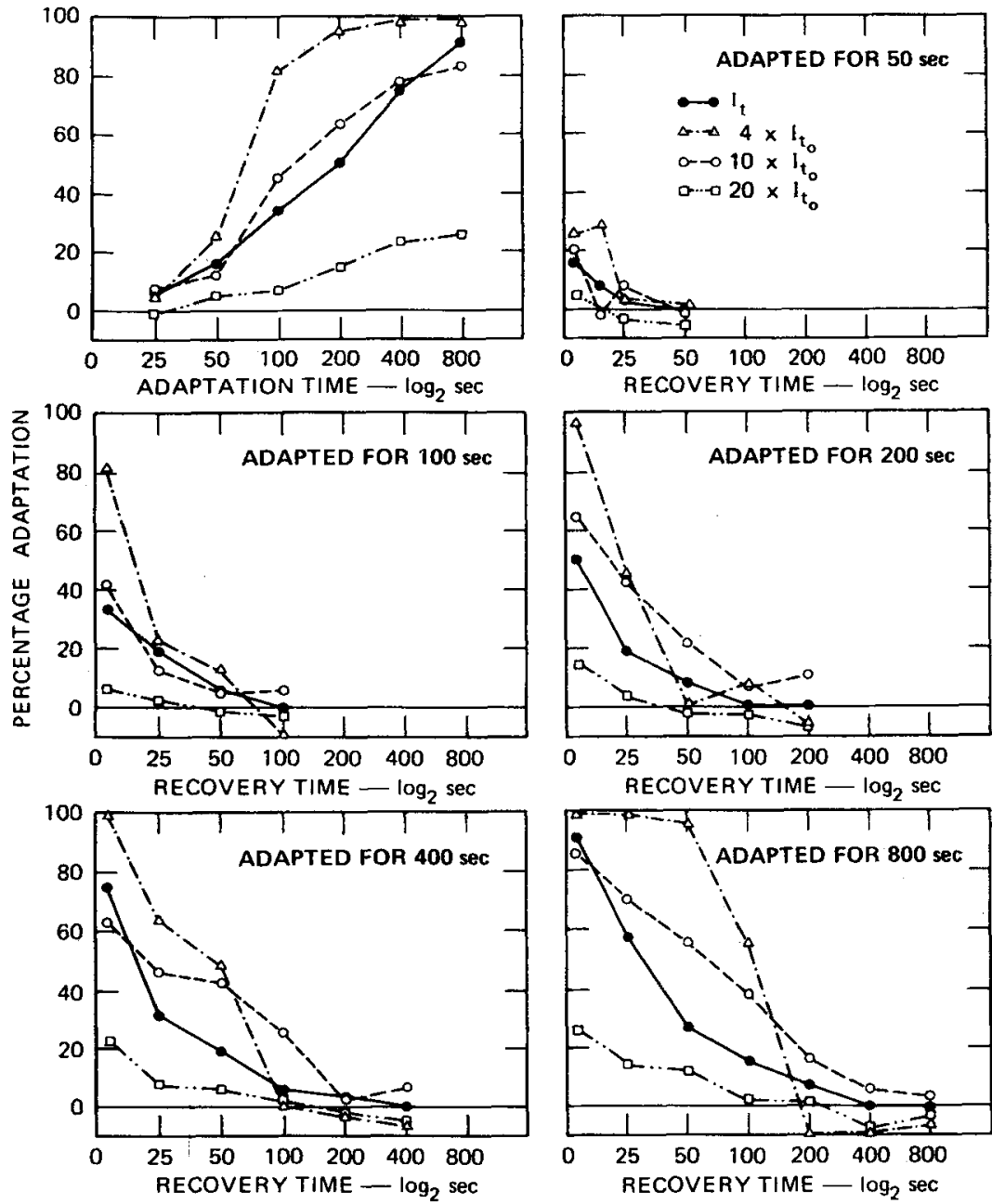

Fig. 4. Comparison of changes in $I_{t}$ and in subjective intensity of 4,10 , and $20 \times I_{t_{0}}$ as a function of self-adaptation to $10 \times I_{t_{o}}$ of methyl isobutyl ketone and subsequent recovery. The course of adaptation as a function of $\log _{2}$ time is shown in the upper left graph. Recovery is shown separately for each adaptation time as a function of $\log _{2}$ time in the other graphs.

exponent increased rather markedly with increased adaptation time. Cain and Engen (1969) observed a similar increase in exponent at increasing concentrations of the adapting stimulus.

\section{DISCUSSION}

The purpose of the present experiment was to obtain a direct comparison of changes in $I_{t}$ and in the subjective intensity of suprathreshold stimuli during the course of olfactory adaptation and recovery. The combined method used in this experiment provided information as to changes in $I_{t}$ and subjective intensity of suprathreshold stimuli during each test session, thus allowing such a direct comparison. In addition, it was possible to estimate the effect of various periods of adaptation on the psychophysical power function.
The threshold detection procedure has been used by Stuiver (1958) and ourselves (Steinmetz et al, 1970) in the past, with considerable success. It is an objective measure and is sensitive to small changes in $I_{t}$. However, as used in those earlier experiments, the adaptation was discontinuous, being interrupted periodically by the insertion of test stimuli, during which time some recovery could occur. In the present experiment we minimized this possible source of artifact by using only one test period during each adaptation session. This variation allowed a more precise artifact-free estimation of the change in $I_{t}$ as a function of adaptation time.

To obtain estimates of changes in subjective intensity in this experiment, we used an unstructured magnitude-estimation procedure that was not dependent on sensorial memory for any particular standard. The results indicated that this procedure can be used reliably to very good advantage in experiments of this kind. Ss can apparently develop an internal scale of subjective intensity that is quite stable for weeks. This procedure is to be contrasted with those using a modified category. scale (Stone, 1966; Steinmetz et al, 1970) and cross-modal matching (Ekman et al, 1967).

In the procedure using a modified category scale, Ss were given a standard and asked to assign it the maximum value possible (they could be asked to assign it any other value, but the criticism would still remain). Thus, limits were established that could not be exceeded. Moreover, it required $S$ to remember the subjective intensity of the standard over a prolonged period in an environment where it was changing continuously. In addition to possible response bias (i.e., decreasing values with increasing adaptation time), the procedure allowed only limited discontinuous scale values. Ekman et al (1967) avoided some of these objections by using a cross-modal matching procedure in which finger span was scaled independently of perceived odor intensity. This modification provided a continuous measure and, as they have shown, seems to be quite reliable over time. However, their procedure also used a standard reference stimulus that set an initial upper limit for the subjective intensity of any subsequent stimulus. And, therefore, as they point out, quantitative comparisons of the course of adaptation to stimuli of differing concentrations could not be made. The present use of an unstructured magnitude-estimation procedure seemed to overcome most of these objections and was reliable and easy to use.

In our opinion, the method of choice for studies of olfactory adaptation and recovery would be a combined method such as that used in the present experiments. Although the method is time-consuming, the increase in information gained over a wide range of the olfactory spectrum seems well worth the additional effort since it is not possible, a priori, to predict changes in subjective intensity of suprathreshold stimuli from changes in $I_{t}$ or vice versa. Use of this combined procedure with different classes of odorants and different concentrations of the adapting stimuli during self- and cross-adaptation may provide a scheme that would be useful in establishing a rational system for odor classification.

An aspect of the data that deserves special comment is the increase in the exponent of the psychophysical power 


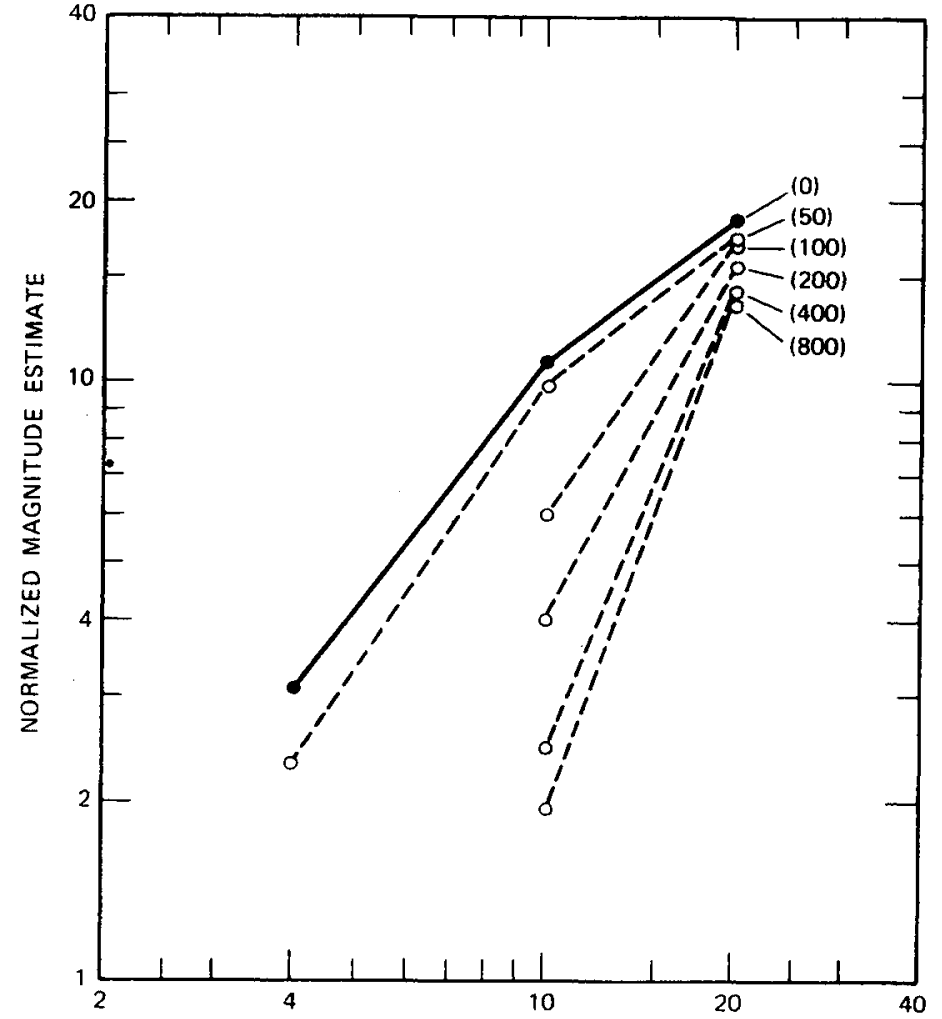

STIMULUS CONCENTRATION - multiples of $t_{t_{0}}$ (adaptation time in parentheses)

Fig. 5. Average, normalized psychophysical power functions for methyl isobutyl ketone as a function of self-adaptation to $10 \times I_{t_{0}}$.

function observed after adaptation. Cain and Engen (1969) reported an increase in the exponent with increasing concentrations of the adapting stimulus for two odorants when the adaptation period consisted of three breaths. They were able to show only minor effects of increasing the duration (number of breaths) of adaptation. Our results, on the other hand, indicated a substantial effect of duration of adaptation on the exponent when the adaptation times ranged from 25 to $800 \mathrm{sec}$, considerably longer than those their results and those of th present experiment provide strong evidence that as adaptation proceeds, the exponent of the psychophysical power function increases in some systematic way.

We would like to suggest that this effect can be at least partially explained in terms of Weber's law, which states that a just noticeable difference (ind) in subjective intensity of a stimulus is a function of the absolute concentration of the stimulus. Applied to the present case, this would mean that a longer adaptation time or a higher adapting concentration would be necessary to reduce the subjective intensity of a stimulus one jnd if the test stimulus were high than if it were low. Since $I_{t}$ is also increasing rapidly, the effective range is reduced accordingly. The consequent effect on the power function would be to increase its slope in $\log -\log$ coordinates, which is what has been observed.

\section{REFERENCES}

CAIN, W. S., \& ENGEN, T. Olfactory adaptation and the scaling of odor intensity. In $C$. Pfaffman (Ed.), Olfaction and taste. New York: Rockefeller Press, 1969. Pp. 127-141.

EKMAN, G. BERGLUND, B., BERGLUND, U. \& LINDVALL, T. Perceived intensity of odor as a function of time of adaptation. Scandinavian Journal of Psychology, 1967, 8, 177-186.

STEINMETZ, G., PRYOR, G. T., \& STONE, H. Effects of blank samples on absolute odor threshold determinations. Perception \& Psychophysics, 1969, 6, 142-144.

STEINMETZ, G., PRYOR, G. T., \& STONE, H. Olfactory adaptation and recovery in man as measured by two psychophysical techniques. Perception \& Psychophysics, 1970, 8, 327-330.

STONE, $H$. Factors influencing the behavioral responses to odor discrimination-A review. Journal of Food Science, 1966, 31, 784-790.

STONE, H., \& BOSLEY, J. J. Olfactory discrimination and Weber's law. Perceptual \& Motor Skills, 1965, 20, 657-665.

STONE, H., OUGH, C. S., \& PANGBORN, R. M. Determination of odor difference thresholds. Journal of Food Science, 1962, 27, 197-202.

STONE, H., PRYOR, G., \& STEINMETZ, G. The design and operation of an improved olfactometer for behavioral and physiological investigation. Behavior Research Methods \& Instrumentation, 1969, 1, 153-156.

STUIVER, M. Psychophysics of the sense of smell. Thesis, University of Groningen, 1958.

\section{NOTES}

1. This research was supported by Grant No. NB 07832 from the National Institute of Neurological Sciences of the National Institutes of Health. The authors thank the panel members for their cooperation.

2. Address: Life Sciences Division, Stanford Research Institute, Menlo Park, California $\mathbf{9 4 0 2 5 .}$

(Accepted for publication June 22, 1970). 